\title{
Gartenstadt Schweiz - Großdorf oder Metropole?
}

\section{Vom Mittelland zur «Stadt Schweiz»}

Das Schweizer Mittelland zwischen Genfersee und Bodensee ist ein metropolitaner Raum, eine locker gebaute Gartenstadt mit rund fünf Millionen Einwohnern und einer beachtlichen Wirtschaftskraft. Zwei Jahrzehnte einer rasanten ökonomischen und technischen Entwicklung haben dazu geführt, daß die einst «idyllischen» Kleinstädte und Dörfer zu einem Wirtschafts- und Lebensraum zusammengewachsen sind - zumindest wenn man die Aktionsräume, die alltäglichen Funktionsabläufe von Herrn und Frau Schweizer zugrunde legt und die Siedlungs- und Verkehrsstrukturen betrachtet. Der Volksmund bringt diesen Trend mit dem Spott "Züri West» (für Bern) treffend auf den Punkt.

Die offizielle Abgrenzung der Agglomerationen (BFS 1994, vgl. Abb. 1) wie auch eine Analyse der Pendlerverflechtungen zeigen es deutlich genug: die herkömmliche Auffassung von autonomen, sauber voneinander getrennten Städten und Agglomerationen mit Kernstadt und Vorortsgemeinden ist überholt. Nicht nur ist der "ländliche Zwischenraum» zwischen den Agglomerationen verschwunden, sondern die Schlafgemeinden der $60 \mathrm{er}$ und $70 \mathrm{er}$ Jahre haben kräftig an wirtschaftlicher Bedeutung, an Arbeitsplätzen zugelegt; der Agglomerationsgürtel ist der am stärksten wachsende Siedlungstyp, und zwar nicht mehr nur in bezug auf die Bevölkerung. Daraus ergibt sich auch, daß die dezidierte Zuordnung der Pendlereinzugsbereiche allmählich einem unübersichtlichen Geflecht von Beziehungen weicht.

\section{Agglomeriten und A-Städte}

Es wäre nun allerdings nicht zutreffend anzunehmen, daß diese "Mittellandstadt Schweiz» ein homogenes Ganzes bildete - das genaue Gegenteil ist richtig. Nicht der vielzitierte Röschtigraben trennt das Land, sondern das Gefälle zwischen der sichtbaren und der unsichtbaren Stadt: Die «Agglomeriten» verlassen die unwirtlichen Kernstädte und ziehen sich zum Wohnen in die scheinbar heile Welt der prosperierenden Landgemeinden zurück. Sie entfalten indessen ihre Arbeits-, Ausbildungs-, Konsum- und Unterhaltungsaktivitäten weiterhin in den Zentren; damit profitieren sie vom attraktiven städtischen Angebot, ohne aber die urbane Kultur mit ihrer Offenheit, Vielfalt und Widersprüchlichkeit mitzutragen und ohne Verständnis für die sich verschärfenden Pro- bleme in den Kernstädten. Der - realisierte! - Traum vom Einfamilienhaus ist denn auch (psychologisch betrachtet) eine Dissoziation des Städtischen, eine Zuflucht in der Geborgenheit einer agrarischen Vergangenheit. Dank hoher Mobilität sind solche inkonsequenten Abspaltungsprozesse bzw. das Nebeneinander von zwei Wirklichkeitssphären möglich geworden, allerdings um den Preis steigender sozialer und ökologischer Kosten. Dazu gehört die Mobilitätsfalle: Die Agglomerationsgürtel sind locker gebaut (keineswegs Verdichtungsgebiete), und sie weisen deshalb hohe relative Erschließungskosten auf; ÖV-Netze sind weitmaschig, großenteils schlecht ausgelastet und folglich unrentabel. Die Kombination (lockere Siedlungsstruktur) $\times$ (städtische Lebensweise) schlägt sich damit als Zwangsmobilität nieder, Autofahren wird zur Existenzgrundlage.

In den Kernstädten setzt dadurch ein doppelter Belastungsprozeß ein (vgl. Abb. 2): Die steigenden Ansprüche an uneingeschränkte automobile Erreichbarkeit überfordern das Verkehrssystem selbst, und die so generierten Umweltbelastungen und städtebaulichen Destruktionsprozesse beeinträchtigen die Qualität städtischer Standorte. Die sinkende Wohnqualität führt zu einer zunehmend selektiven Bevölkerungsentwicklung, zu einer "Stadt ohne Kinder» und zu einer Entmischung entlang von Einkommens- und Mobilitätsgradienten: Ausländer, Alte, Arbeitslose bleiben zurück in diesen A-Städten - kurz: die andern, die keinen Platz haben im ordentlichen Weltbild der erfolgsgewohnten Agglomeriten. Dazu kommen Sicherheits- und Fürsorgeprobleme, der Erneuerungsbedarf der städtischen Infrastrukturen, die Lasten der zentralörtlichen Einrichtungen vom Theater bis zum Frauenhaus, die aufwendige Komplexität innerstädtischer Bauvorhaben, und last but not least die vordringliche Frage, welcher Grad und welche Form der Erreichbarkeit, der Mobilität (ÖV, MIV) für die Kernstädte möglich sei und wer dafür bezahle.

Alsbald setzt dann der bekannte Teufelskreis ein: Bei den städtischen Finanzen öffnet sich die Schere zwischen schwindenden Einnahmen und steigenden Verpflichtungen, und sie öffnet sich immer weiter beim Versuch, in einer Flucht nach vorn eine städtische Implosion zu

Martin Boesch, Prof. Dr., Forschungsstelle Wirtschaftsgeographie und Raumplanung HSG, Postfach 67, 9011 St. Gallen 


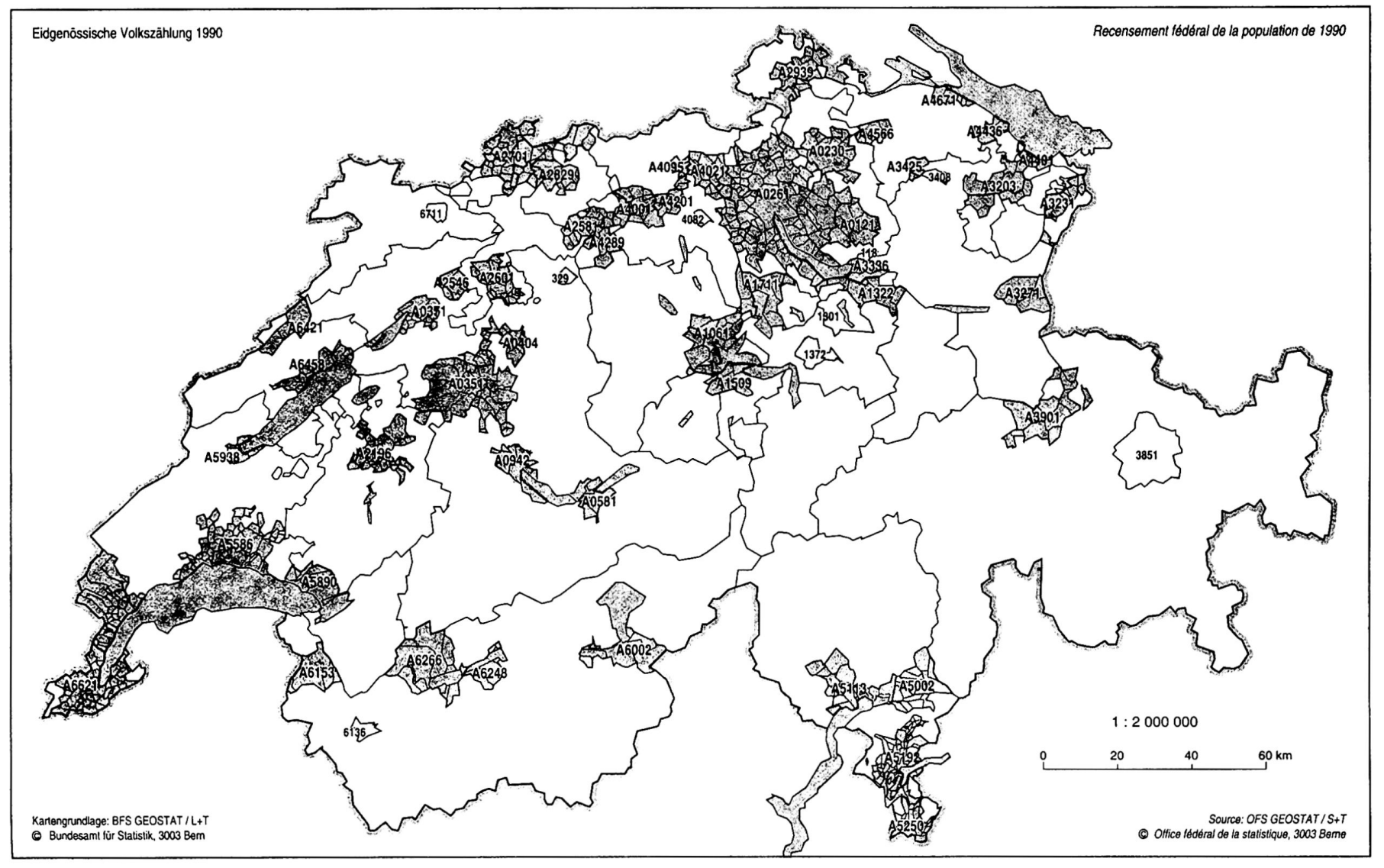

Abb.1 Die Stadt "Schweiz" zwischen Genfersee und Bodensee 


\section{Räumliche Segregations-Prozesse}

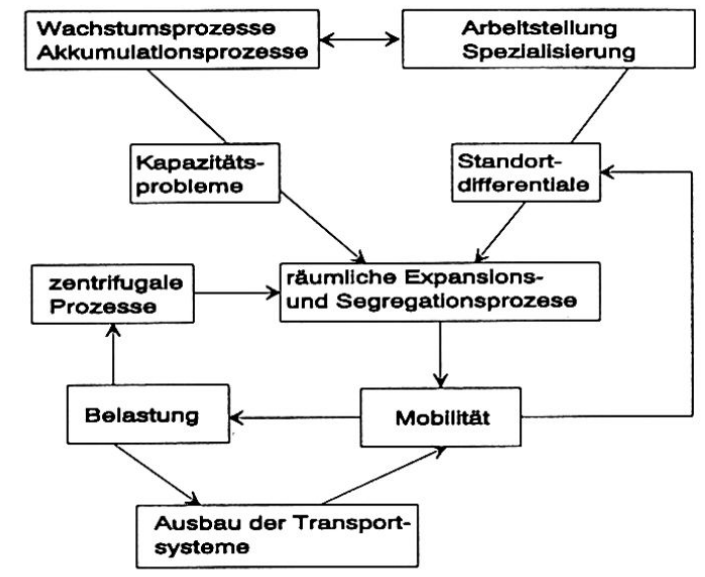

Abb. 2 Räumliche Segregationsprozesse (Entwurf M. Boesch).

erzeugen. Die wirtschaftliche Dynamik soll verstärkt auf die Stadtmitte fokussiert werden, die Nutzungsdichte weiter erhöht werden um den Preis einer immer großräumigeren Entmischung. Es ist offensichtlich: «autonome» Insellösungen sind untaugliche Ansätze.

\section{Regionale Kooperation}

Der Öffnung der Problemfelder über kommunale und kantonale Grenzen hinaus kann nur durch regionale Kooperation begegnet werden. Der Verlust des Kongruenzprinzips, das heißt der Kongruenz von Problemraum und Handlungsraum, muß überwunden werden. Was von der Gründerzeit bis in die 20er Jahre die Eingemeindung der Vororte brachte, muß heute allerdings mit andern Ansätzen realisiert werden: Die sachlich und zeitlich variable "Geometrie» der Aufgaben verhindert das Ziehen fester Grenzen. Statt dessen müssen neue Formen föderalistischer Zusammenarbeit und gemeinsamer Aufgaben- und Lastenteilung entwickelt werden, problembezogen über alle Stufen hinauf von den Gemeinden bis zum Bund.

Regionale Kooperation meint aber nicht einfach noch mehr Zweckverbände; vielmehr geht es um nichts Geringeres als urbane Kohärenz. Das bedeutet die Notwendigkeit, daß sich die Stadtidee, urbanes Denken über die zu eng gewordenen Grenzen der Kernstädte hinaus aufs "Land», in die Agglomerationen hinaus ausbreitet. Nur wenn sich diese als Teile der Stadt, als Quartiere verstehen, mit ihrem Eigenleben, ihren Eigenheiten, aber doch als Teil einer größeren Gemeinschaft, kann es gelingen, auf der Basis städtischer Solidarität die anstehenden Aufgaben zu lösen.
Eine Art Wahrnehmungsbarriere blockiert aber einstweilen solche Ansätze, die Kernstädte werden mit ihren Problemen allein gelassen: Der faktische Verstädterungsprozeß $ß$ des ganzen Mittellandes ist im soziokulturellen und politischen Verständnis seiner Bevölkerung noch nicht nachvollzogen. Die Agglomerationsgemeinden sehen sich immer noch als Dörfer, betonen ihre Autonomie, ihre ländlichen Qualitäten und ihre bäuerlichgewerbliche Politkultur. Mit dem Rücken zum Zentrum sagen sie: «Was gehen uns die Städte an?» und versuchen, ihren stärksten Trumpf, die noch großen, offenen Bauzonen, möglichst rasch und locker einzusetzen.

Kantone und Bund verstärken diese Indifferenz: Sie agieren so, als ob Kernstädte und Agglomerationen Gemeinden wie alle andern wären (was rechtlich gesehen in den meisten Fällen auch stimmt), und dort, wo Sonderfälle auftreten (z. B. mit den Maßnahmeplänen zum USG), verwehren sie ihnen das nötige Vorgehen. Dabei sind es gerade die Städte, die beim Vollzug von Bundesrecht besonders gefordert sind (so auch in der Drogenpolitik, in der Sozialpolitik). Und umgekehrt sind nach wie vor die Städte die dynamischen Pole wirtschaftlicher und soziokultureller Innovation. Um dieser Herausforderung gerecht zu werden, bedürfen Kantone und Bund einer dezidiert urbanen Dimension in ihrer Politik; sie müssen den Schritt machen von einer Land-Schweiz zur StadtSchweiz.

\section{Die neue Stadtpolitik}

Der Schritt vom Großdorf zur Metropole ist also zuallererst eine Frage der Perspektive, der Optik: Wie verstehen wir uns? Es ist bemerkenswert, daß die Raumordnungspolitik mit den «Grundzügen» diese Diskussion lanciert hat: die Schweiz als "vernetztes Städtesystem». Richtig ausgeformt könnte so die Gratwanderung zwischen Wettbewerbsfähigkeit, urbaner Lebensqualität und Nachhaltigkeit gelingen. Nur vage angesprochen ist allerdings die Zukunft des Alpenraumes, und umstritten ist nach wie vor die Gestaltung des Außenbereiches - Kooperationsmechanismen sind auch hier nötig. Eng verknüpft mit der Regionalpolitik muß folglich die Reform des horizontalen und vertikalen Finanz- und Lastenausgleichs gesehen werden, und zwar auf allen Stufen. Dabei kann es nicht nur um eine - dringend nötige - sektorale Effizienzsteigerung gehen; gefordert ist vielmehr auch eine bessere ressortübergreifende Zielkongruenz der unterschiedlichen Akteure.

Neben dem Bund sind auch die Kantone gefordert: Gefragt ist eine agglomerations- oder regionsorientierte Ausrichtung der kantonalen Politik anstelle der bisherigen gemeindeorientierten; eine konzeptionelle Ausrichtung auf überkommunale (auch Kantonsgrenzen überschreitende) Zusammenarbeit, ein diesbezüglich stetes Anmahnen der Gemeinden durch den Kanton. Die Stadtregionen übernehmen Kompetenzen, aber auch Eigenverantwortung. Dies gilt vor allem (aber nicht nur) im Bereich der Siedlungs- und Verkehrspolitik. Erste 
Ansätze wie die Koordination von Siedlungs- und S-Bahn-Konzepten im Kanton Bern oder gar das vorgesehene Agglomerationsgesetz im Kanton Freiburg sind Schritte in die richtige Richtung. Angesichts der hohen Mobilität wird aber auch zum Beispiel in der Sozialpolitik eine Kompetenzverschiebung von der Gemeinde auf die Stadtregion notwendig.

Damit das «vernetzte Städtesystem» konkrete Gestalt annehmen kann, müssen unsere Siedlungsstrukturen viel kompakter werden, und sie müssen viel entschiedener auf die ÖV-Netze ausgerichtet sein. Gleiches gilt auch für öffentliche Institutionen und publikumsintensive Betriebe. Die Koordination von ÖV-Knoten und Zentren (traditionellen und vielen neuen) ist ein wichtiger Beitrag zur wirtschaftlichen Stärkung der Standorte. Dazu gehören eine attraktive städtebauliche Gestaltung und die Komplementarität vielfältiger städtischer Funktionen und Nutzungen.

Kompaktere Siedlungsstrukturen werden gefördert durch die Verdichtung nach innen; sie basiert auf einer Anhebung der minimalen Ausnützung und einer qualifizierten Anforderung an die Baureife: Hinreichend erschlossen ist in Zukunft eine Parzelle nur im Fußgängerbereich von Zentren und/oder ÖV-Knoten mit 1/4-Stunden-Takt. Denn nur so kann die Mobilität der Zukunft nämlich eine Stadt der kurzen Wege im Umweltverbund - sichergestellt werden. Der Regionalverkehr muß als Aufgabe von den Stadtregionen getragen werden; sie werden rasch lernen, mit der Kostenwahrheit umzugehen. Effizienzsteigerung ist auch angesagt bei andern öffentlichen Versorgungs- und Dienstleistungen, soweit sie nicht privatisiert und damit ohnehin entsprechend ausgerichtet werden. Damit wird der Trend zu kompakten Siedlungen weiter unterstützt.

Um ihre Wettbewerbsfähigkeit zu verbessern, werden die Stadtregionen ihre Aufmerksamkeit vermehrt auch der Wohnqualität zuwenden. Die Wohnbauförderung wird sich stärker auf den Erneuerungs- und Sanierungsbedarf einstellen müssen, und die jetzt in Kernstädten angelaufenen Maßnahmen im Wohnumfeld wie Verkehrsberuhigung und Aufwertung der öffentlichen Räume werden auch in Siedlungen aktuell, die sich jetzt noch für ländlich halten, weil gerade auch dort der Bedarf ausgewiesen ist.

Unterstützt von vielen andern Dimensionen einer städtisch ausgerichteten regionalen und kantonalen Politik, wird so der Umbau vom Großdorf zur Stadt Schweiz vollzogen - gerade rechtzeitig für unsere Öffnung Richtung Europa!

\section{Literatur}

BFS - Bundesamt für Statistik (Hrsg.)(1994): Eidg. Volkszählung 1990. Die Raumgliederungen der Schweiz, Bern.

BOESCH, M. (1982): Zur Bestimmung von Verdichtungskernräumen in der Schweiz. In: ARL (Hrsg.): Studien zur Abgrenzung von Agglomerationen in Europa, Hannover.

BOESCH, M. (1992): Urbanität - was die Stadt zusammenhält. In: LANZ, U. (Hrsg.): Gallusstadt 1992. Jahrbuch der Stadt St. Gallen, pp. 6-21.

BRP - Bundesamt für Raumplanung (1994): Grundzüge der Raumordnung Schweiz, Bern.

BSP - Bund Schweizer Planer (Hrsg.) (1996): Grenzüberschreitend Planen + Handeln (= Collage 1/96).

BUCHMÜLLER/FINGERHUTH/HUBER (Hrsg.) (1993): Management der postmodernen Stadt (= ORL-Bericht 85), Zürich.

FREY, R. L. (1990): Städtewachstum - Städtewandel. Eine ökonomische Analyse der schweizerischen Agglomerationen, Basel.

FREY, R. L. (1996): Stadt: Lebens- und Wirtschaftsraum, Zürich.

GABATHULER et al. (1990): Siedlungsbegrenzung Schweiz - Möglichkeiten und Grenzen einer Siedlungsentwicklung nach innen (= NFP Boden 57), Liebefeld BE.

GÜLLER, P./BREU, T. (1996): Städte mit Zukunft - ein Gemeinschaftswerk. Gesamtbilanz des NFP Stadt \& Verkehr, Zürich.

HAUSMANN, U. (1996): Innovationsprozesse von produktionsorientierten Dienstleistungsunternehmen und ihr räumlicher Kontext. Ein akteurbezogener theoretischer Bezugsrahmen, entwickelt am Beispiel von London und Zürich. Diss. HSG, Bamberg.

HAUSMANN, U./EGGER, U. (1995): Strukturwandel und produktionsorientierte Dienstleistungen. Analyse des schweizerischen Städtesystems zwischen 1975 und 1991. In: DISP 122/14-19, Zürich.

HÜTTENMOSER, M./DEGEN-ZIMMERMANN, D. (1995): Lebensräume für Kinder. Empirische Untersuchungen zur Bedeutung des Wohnumfeldes für den Alltag und die Entwicklung der Kinder (= NFP Stadt \& Verkehr 70), Zürich.

KLOTI, U., et al. (1993): Agglomerationsprobleme und vertikale Zusammenarbeit. Umweltschutz und öffentlicher Verkehr in den Großagglomerationen Lausanne und Zürich (= NFP Stadt \& Verkehr 49), Zürich.

KNOFLACHER, H. (1993): Zur Harmonie von Stadt und Verkehr, Wien.

MEIER-DALLACH, H.-P./HOHERMUTH, S. (1994): Stadtkultur im Großdorf Schweiz (= NFP Stadt \& Verkehr 47), Zürich.

MUMFORD, L. (1961): Die Stadt. Geschichte und Ausblick (= The City in History), München.

REGIERUNGSRAT BE (1992): Bericht des Regierungsrates an den Großen Rat: Berner S-Bahn/Siedlung, Bern.

REY, A. (1993): Finanzreformen für die Schweiz, Solothurn.

STÄHLIN-WITT, E./BLÖCHLINGER, H. (1995): Marktwirtschaftliche Reformvorschläge: unterstützen sie die Raumplanung?, Bern.

STIENS, G. (Hrsg.) (1995): Städtevernetzung. Anknüpfungspunkte - Konzepte - Erfordernisse (= Materialien zur Raumentwicklung 72), Bonn. 